\section{Systematic bias in baroclinic energy estimates in shelf seas} \\ Stephenson, Gordon; Green, Mattias; Inall, Mark E.

\section{Journal of Physical Oceanography}

Published: 01/09/2016

Peer reviewed version

Cyswllt i'r cyhoeddiad / Link to publication

Dyfyniad o'r fersiwn a gyhoeddwyd / Citation for published version (APA):

Stephenson, G., Green, M., \& Inall, M. E. (2016). Systematic bias in baroclinic energy estimates in shelf seas. Journal of Physical Oceanography, 46(9).

\footnotetext{
Hawliau Cyffredinol / General rights

Copyright and moral rights for the publications made accessible in the public portal are retained by the authors and/or other copyright owners and it is a condition of accessing publications that users recognise and abide by the legal requirements associated with these rights.

- Users may download and print one copy of any publication from the public portal for the purpose of private study or research.

- You may not further distribute the material or use it for any profit-making activity or commercial gain

- You may freely distribute the URL identifying the publication in the public portal ?
}

Take down policy

If you believe that this document breaches copyright please contact us providing details, and we will remove access to the work immediately and investigate your claim. 


\section{Systematic bias in baroclinic energy estimates in shelf seas}

Gordon R. Stephenson* and J. A. Mattias Green ${ }^{\dagger}$ and Mark E. Inall ${ }^{\ddagger}$

${ }_{3}^{*}$ Bangor University, School of Ocean Science, Askew St., Menai Bridge, Isle of Anglesey, LL59

${ }_{4} 5 \mathrm{AB}, \mathrm{UK}$

${ }_{5}^{\dagger}$ Corresponding author address: Bangor University, School of Ocean Science, Askew St., Menai

6 Bridge, Isle of Anglesey, LL59 5AB, UK

7 E-mail: m.green@ bangor.ac.uk

${ }_{8}^{\sharp}$ Scottish Association for Marine Science, Scottish Marine Institute, Oban, Argyll, PA37 1QA, UK 


\begin{abstract}
9 A simple model of an internal wave advected by oscillating barotropic flow 10 suggests flaws in standard approaches to estimating properties of the internal 11 tide. When the $\mathrm{M}_{2}$ barotropic tidal current amplitude is of similar size to 12 the phase speed of the $\mathrm{M}_{2}$ baroclinic tide, spectral and harmonic analysis 13 techniques lead to erroneous estimates of the amplitude, phase, and energy in ${ }_{14}$ the $\mathrm{M}_{2}$ internal tide. In general, harmonic fits and bandpass or low-pass filters ${ }_{15}$ that attempt to isolate the lowest $\mathrm{M}_{2}$ harmonic significantly underestimate ${ }_{16}$ the strength of $\mathrm{M}_{2}$ baroclinic energy fluxes in shelf seas. Baroclinic energy ${ }_{17}$ flux estimates may show artificial spatial variability, giving the illusion of 18 sources and sinks of energy where none are present. Analysis of previously 19 published estimates of baroclinic energy fluxes in the Celtic Sea suggests this 20 mechanism may lead some values to be 25 to $60 \%$ too low.
\end{abstract}




\section{Introduction}

Interactions of the barotropic tide with sloping ocean bathymetry in the presence of stratification produce tidal-frequency internal waves, the internal tide, that carry energy to the ocean interior or to the continental margins (see, e.g., Wunsch and Ferrari, 2004 for a review). Where these internal waves break, the result is turbulence, energy dissipation and, potentially, vertical mixing. Internal waves are consequently the main source of dissipation in the abyssal ocean (e.g., Wunsch and Ferrari, 2004), but a significant fraction of the tidal energy in shelf seas is in the internal wave field, with the mode-1 semidiurnal $\left(\mathrm{M}_{2}\right)$ tide responsible for an estimated 20-60 $\mathrm{GW}$ of energy propagating shoreward of the $175 \mathrm{~m}$ isobath and another $40-120 \mathrm{GW}$ dissipating on the continental shelf slope (Kelly et al., 2013). Ocean circulation patterns are sensitive to the global distribution of the resulting vertical mixing (Melet et al., 2013). Locally, breaking internal waves cause vertical mixing that enhances vertical nutrient transport (Sharples et al., 2001) and contributes to the high primary productivity of the shelf break region, indirectly supporting fisheries (Sharples et al., 2007). Internal tides can create strong vertical shear in the water column, which can impact drilling and dredging operations (Osborne et al., 1978), as well as tidal power generation schemes.

Observations of the internal tide in shelf seas reveal many poorly explained features of the wave field. Spectra often show considerable energy at higher harmonics of the semidiurnal $\left(\mathrm{M}_{2}\right)$ tide (Rippeth and Inall, 2002; Robins and Elliott, 2009; Shroyer et al., 2011). In some cases, higher harmonics may be more evident than the fundamental tide or inertial forcing frequency, as shown for higher vertical modes by MacKinnon and Gregg (2003). Futhermore, large spatial and temporal variability in the strength and phase of the internal tide is common: off the coast of New Jersey, Shroyer et al. (2011) observed spatial variability in baroclinic energy fluxes, with both increases and decreases in strength moving from continental slope to shelf. These energy 
fluxes were also concentrated in one or two "pulses" during particular phases of the barotropic tide. This intermittency is often associated with nonlinear internal waves (NLIW). However, as we demonstrate in this article, a linear superposition of barotropic and baroclinic waves can lead to many of the features often associated with NLIW.

Although the generation mechanisms of the internal tide are fairly well understood and the energy conversion rate can be quantified (Green and Nycander, 2013), internal tides have proven difficult to predict, and temporal variability in the internal tide has been hard to explain. Nash et al. (2012) hypothesized that the locally-generated component of the internal tide should have a fixed phase relationship to the local barotropic tide, but that long-distance propagation of the internal tide across ocean basins, through mesoscale variability, results in remotely-generated internal tides with an incoherent phase relationship to the local barotropic tide. Nash et al. (2012) decomposed the internal tide into coherent, locally-generated and incoherent, remotely-generated components, and found that the majority of shoreward propagating energy has a time-varying phase offset relative to the local barotropic tide. They therefore concluded that the internal tide on the New England continental shelf is mostly generated at remote locations. Further results incongruous with local barotropic forcing were seen by Hopkins et al. (2014) and Inall et al. (2000), who saw that baroclinic energy fluxes on the European shelf decreased in strength during the spring tide, when generating forces should be greatest. The distribution of energy over vertical wave modes is also often a mystery; MacKinnon and Gregg (2003) found that the distribution of energy between different vertical modes of the $\mathrm{M}_{2}$ tide varies in time, but with no apparent pattern or coherence.

While the many processes contributing to temporal and spatial variability in the internal tide make internal tide prediction a complicated task, there remains considerable uncertainty in more elementary properties of the wave field. In the Celtic Sea, values of the average onshore baroclinic energy flux, an important sink term in the global tidal energy budget, range from $73 \mathrm{~W} \mathrm{~m}^{-1}$ (Green 
et al., 2008) or $100 \mathrm{~W} \mathrm{~m}^{-1}$ (Hopkins et al., 2014) to as much as $1600 \mathrm{~W} \mathrm{~m}^{-1}$ (Inall et al., 2011), a difference of more than an order of magnitude. In the Celtic Sea, some of the variability in baroclinic energy fluxes has been attributed to the complicated nature of bathymetry at the shelf (Vlasenko et al., 2014) or to changes in propagation across the shelf (Stephenson et al., 2015). Some part of the difference may be due to the positioning of moorings, time of year, or analysis techniques used. Understanding the cause of such a wide spread in observed energy fluxes, and whether the same processes apply to other shelf seas, is vital to understanding global patterns of tidal energy loss and vertical mixing.

The objective of this paper is to examine the implications of barotropic / baroclinic tide interactions for baroclinic energy flux estimates. We show that some of the temporal and spatial variability of the internal tide can be explained by a fairly simple advective process. This wave advection process is a very simple case of the wave-wave interactions examined by Holloway (1983) and Pinkel (2008). Those studies sought to reconstruct the internal wave wavenumber-frequency spectral continuum observed in the open ocean by modeling the effects of wave-wave advection and Doppler "smearing" by oceanic currents on the spectra of an internal wave field constructed of waves of discrete frequencies. In this paper, we consider only interactions between the barotropic tide and one mode of the baroclinic tide; we are interested in the consequences this interaction has on estimates of the strength of the baroclinic tide and energy fluxes. Following Green et al. (2010), we model a sinusoidal internal tide advected by a sinusoidally oscillating barotropic tidal flow. With this linear superposition of a mode- 0 and mode- $n$ wave, we reproduce many of the features of the internal tide described above. Furthermore, we find that, where the barotropic tide is strong, standard analysis techniques and filters may lead to significant underestimates of the strength of the internal tide and of baroclinic energy fluxes. 
Section 2 describes our model of an advected internal wave. Section 3 discusses the features of the model advected wave, while the implications for shelf-seas observations of the tide are discussed in Section 4. Conclusions are in Section 5.

\section{Methods}

We assume that a sinusoidal plane wave of a single frequency propagates at an angle $\theta$ measured clockwise from north. This wave can represent many things: the displacement of the thermocline in a two-layer wave, or the baroclinic velocity at a fixed depth, or anything that is in the form of a linear, sinusoidal wave, and we thus write:

$$
\eta(x, y, t)=A \sin (k \sin (\theta) x+k \cos (\theta) y-\omega t)+B,
$$

where $A$ is the wave amplitude (of, e.g. isopycnal displacement or baroclinic velocity), $B$ is the mean value, $k$ is the horizontal wavenumber, and $\omega$ is the frequency of the wave, in the following assumed to be that of the $\mathrm{M}_{2}$ tide, $2 \pi / 12.42$ hour $^{-1}$. The phase velocity of the internal wave is defined as $c=\omega / k$.

We assume that the internal wave remains sinusoidal when viewed from a reference frame moving with the advective flow. To represent the advection of the propagating wave by the barotropic flow, we replace $x$ and $y$ by $x_{a d v}(t)$ and $y=y_{a d v}(t)$, where

$$
x_{a d v}(t)=x-\int_{t_{0}}^{t} U_{b t}(\tau) d \tau \quad \text { and } \quad y_{a d v}(t)=y-\int_{t_{0}}^{t} V_{b t}(\tau) d \tau
$$

account for advection of the internal wave by barotropic flow $U_{b t}$ in the east-west direction and $V_{b t}$ in the north-south direction. We introduce

$$
x_{r}=\sin (\theta) x+\cos (\theta) y \quad \text { and } \quad U_{r o t}=\sin (\theta) U_{b t}+\cos (\theta) V_{b t}
$$


to represent the coordinates and advective motions projected into the direction of propagation of the wave. In these new coordinates, the expression for the advected wave is simplified to

$$
\eta\left(x_{r}, t\right)=A \sin \left(k\left(x_{r}-\int_{t_{0}}^{t} U_{r}(\tau) d \tau\right)-\omega t\right)+B .
$$

In a coordinate system moving with the component of barotropic flow in the direction of wave propagation, say $x^{\star}$, where

$$
x^{\star}\left(x_{r}, t\right)=x_{r}-\int_{t_{0}}^{t} U_{r}(\tau) d \tau=\sin (\theta) x_{a d v}+\cos (\theta) y_{a d v},
$$

the equation has the familiar form

$$
\eta\left(x^{\star}, t\right)=A \sin \left(k x^{\star}-\omega t\right)+B .
$$

In the transition to a stationary coordinate system, as at a mooring where $x$ and $y$ are fixed, the observed wave has a different expression as a function of time. For a semidiurnal tide, the barotropic tidal velocity is represented as $U_{r}=U \cos (\omega t)$, where $U$ is amplitude of the tide projected into the wave propagation direction. The waveform observed at a fixed (Eulerian) point in space, $\eta_{0}$, can be expressed as

$$
\eta_{0}(t)=A \sin \left(\frac{U}{c} \sin (\omega t)-\omega t+\Phi_{0}\right)+B .
$$

The observed waveform is strongly dependent on the barotropic/baroclinic phase difference $\Phi_{0}$, which is a function of $x_{r}$ (distance along the direction of wave propagation). Therefore, the observed wave and its energy and spectral characteristics also vary in $x_{r}$. The phase offset between the barotropic tide and the mode- $n$ baroclinic tide can be written

$$
\Phi_{0}=\left(k_{n}-k_{0}\right) x_{r}
$$

where $k_{n}$ is the horizontal wavenumber of the mode- $n$ internal tide and $k_{0}$ is the horizontal wavenumber of the barotropic tide. $k_{0}$ is generally much smaller than $k_{n}$, so $\Phi_{0} \approx k_{n} x_{r}$. A $\pi$ 
radian difference in $\Phi_{0}$ corresponds to $\sim \frac{1}{2} \lambda_{n}$, where $\lambda_{n}$ is the horizontal wavelength of the $n^{\text {th }}$ mode internal tide.

The physics at work are similar in nature to a Doppler shift; the observed frequency changes as a result of the wave moving relative to the observer. The key difference is that the frequency of motion is close to that of the wave being observed. In a standard Doppler shift, $\omega_{\text {shifted }}=\omega_{\text {true }} \pm U k$, but this formulation is insufficient to describe the observed waveform when the wave and advective flow have similar frequencies. As we shall see, in this case, the frequency shift occurs in discrete steps - to multiples (harmonics) of $\omega$. If there is no barotropic flow (i.e., if $U=0$ ), our case reduces to that of a purely sinusoidal wave with all its energy at the $\mathrm{M}_{2}$ frequency. Because the barotropic flow is vertically uniform and purely horizontal, we can describe a baroclinic tide propagating in the vertical and horizontal directions as a set of stacked horizontally propagating waves, each at a phase offset from the one above. As it is advected, under our simplifying assumptions, the tidal beam retains its shape, and the effects on each layer can be computed independently using the appropriate phase as a function of depth. For the same reason, vertically well-resolved measurements will not mediate the effects of the barotropic advection on the observed waveforms.

\section{Results}

\section{a. Waveforms}

Although the model wave is sinusoidal in $x^{\star}$ and $t$ (Equation 2), Eulerian measurements of the wave (represented by $\eta_{0}$ in Equation 2) are not sinusoidal (Figure 1). Two parameters govern the observed waveform; $\Phi_{0}$, the phase offset between the baroclinic and barotropic wave; and $U / c$, the amplitude of the barotropic flow normalized by the internal wave speed. The observed waveform retains its 12.42 hour periodicity, but exhibits several unusual features that vary with 
the normalized barotropic flow speed, $U / c$, as well as the phase offset between the baroclinic and barotropic flows, $\Phi_{0}$. At small values of $U / c$, these features are limited to a steepening of the wave crest. At larger values of $U / c$, the observed waveform appears more nonlinear, whereas multiple wave crests advected past the observing platform during one tidal period appear as higher frequency signals. Other shapes are possible: at certain phase-offsets, the waves in Figure 1 look like low-frequency solitons (see, e.g., Figure $1 \mathrm{~b}$,f). In this example, $U / c \approx 2$, and the trough or crest is evident for only about 3 hours. One of these waves appears as a wave of elevation (Figure 1f); the other, at a phase offset $\pi$ radians different from the first, is a wave of depression (Figure 1c).

The criteria that $U / c$ be large is equivalent to the flow having a large tidal excursion length scale. With a large tidal excursion, many crests and troughs may be advected past a fixed observer. An important note, though, is that this ratio is a property of the internal wave being considered. Higher vertical modes are slower and have shorter horizontal wavelengths than lower vertical modes. In uniform stratification, for example, the phase speed of the $n^{\text {th }}$ vertical mode, $c_{n}$, scales inversely with mode number $n$, therefore $U / c_{n}$ scales linearly in $n$. Higher vertical mode waves therefore are likely to exhibit a greater degree of distortion as a result of advection.

\section{b. Average values}

Waves are often identified as perturbations to the mean state of some property of the ocean. For example, if $h(t)$ represents the depth of the thermocline, $h(t)$ might be decomposed into $h(t)=$ $\overline{h(t)}+h^{\prime}(t)$, where $h^{\prime}$ is the perturbation associated with a wave and the overbar indicates averaging over some integer number of wave periods. For a linear internal wave, $\overline{h^{\prime}}=0$. When $U / c>0$, however, the time-average of wave properties observed at a fixed location may be non-zero (as in Figure 1), leading to an non-zero offset in the average observed thermocline depth $\overline{h^{\prime}}$. This bias 
term is a function of both $\Phi_{0}$ and $U / c$ (Figure 2). The maximum value of the offset, or the bias in $\overline{h^{\prime}}$, is $\sim 0.6 \mathrm{~A}$ for $U / c \approx 1.7$, where $A$ is the internal wave amplitude. For example, a moored sensor in a flow similar to of Figure $1 \mathrm{~b}$ would observe a wave crest most of the time, followed by a brief downward excursion as the wave trough is swept past by the barotropic flow. A time average of measurements at this phase-offset is therefore biased towards the value at a wave crest; this is true for any observation window over an integer number of wave periods. Half a wavelength away (half a wavelength of the internal tide), the opposite bias is observed: the time-average is biased towards the wave trough value, to a minimum value of $\overline{h^{\prime}} \approx-0.6 A$, as shown in Figure 1f. For values of $U / c$ increasing beyond 1.7 , more wave crests are swept past the measurement platform in one tidal period, and therefore $\bar{h}^{\prime}$ tends to 0 as $U / c$ tends to infinity.

This bias has consequences. If we assume that this is a 2-layer wave, then variation of $\bar{h}$ with $\Phi_{0}$ will appear as horizontal variations of the mean isopycnal depth, or, equivalently, as a horizontal density gradient. In a linear internal wave affected by the earth's rotation, the zonal velocity perturbation $u^{\prime}$, vertical displacement $h^{\prime}$, and pressure perturbation $p^{\prime}$ are $\pi / 2$ radians out of phase with the meridional velocity perturbation $v^{\prime}$. If $\overline{h^{\prime}}$ is at a maximum (as in Figure $1 \mathrm{~b}$ ), then $\overline{v^{\prime}}=0$ ( $\pi / 2$ radians offset), but $\overline{u^{\prime}}$ is also at a maximum. The depth-averaged flow remains zero for baroclinic motions, and there is therefore no net mass flux as a result of the advective interaction. However, in the upper and lower layers, $\overline{u^{\prime}} \neq 0$ corresponds to time-averaged across-shore flow. At these locations, a mooring will observe time-averaged across-shelf flow in each layer. The direction of across-shelf flow reverses every half-wavelength. On the other hand, if $\overline{h^{\prime}}=0$, then $\overline{u^{\prime}}=0$, but $\overline{v^{\prime}}$ is at a maximum or minimum, corresponding to time-averaged along-shore flow. Assuming the phase offset $\Phi_{0}$ is constant in time at a given location, as for locally-generated internal waves (Nash et al., 2012), the bias cannot be removed by extending the averaging time interval. 


\section{c. Perturbations}

As we have shown, where the barotropic and baroclinic waves interact, the observed average may be biased. If perturbations, $\eta^{\prime}(t)$ are calculated using the observed average, $\overline{\eta_{0}}$, as $\eta^{\prime}(t)=$ $\eta_{0}(t)-\overline{\eta_{0}}$, this bias is directly transferred to perturbation quantities. In the example in Figure $1 \mathrm{~b}$, the average observed value of the isotherm depth is $0.6 A$; therefore, $h^{\prime}$ ranges from $-0.4 A$ to $1.6 A$. Similar conclusions hold for $u^{\prime}, v^{\prime}$, and $p^{\prime}$.

In computing the baroclinic energy fluxes, we calculate $F=u^{\prime} p^{\prime}$. If the perturbation quantities are biased, the range of $F$ increases. In the case of relatively weak advection $(U / c=0.2)$, the maximum value of the flux observed increases by only $\sim 15 \%$, and at a given location the timing of baroclinic energy flux "pulses" is a function of the phase of the IW (Figure 3c). In contrast, when $U / c \approx 1.7, \eta^{\prime}$ ranges from -0.4 to 1.6 times its usual value. Therefore $F$ ranges from 0.2 to 2.56 times its 'actual' value. This serves to exaggerate non-linearity and intermittency in energy fluxes, which appear concentrated in a narrow time interval (Figure 3a). Furthermore, in this case, the peak baroclinic energy flux occurs at a particular phase of the barotropic tide (Figure 3b), while the phase of the IW contributes very little to the timing. The arrival time of a pulse of baroclinic energy is nearly uniform in $x_{r}$.

Tidally-averaged values $(\langle\rangle)$ of $F$ are also affected (Figure 4). As $U / c$ increases from $0,\langle F\rangle$ decreases, to a minimum of 0.5 times its value for the case of no advection. For $U / c>2$ or 3 (depending on $\Phi_{0}$ ), $\langle F\rangle$ increases to a maximum of $\sim 1.3$ times its value for the case of no advection when $U / c \approx 3.3$. The magnitude of the decrease or increase is dependent on $\Phi_{0}$, and can be significant for relatively low values of $U / c$. For example, for $U / c \approx 0.6$, a decrease of up to $15 \%$ in the tidally-averaged fluxes is possible. It should be noted that this decrease is not 
a result of filtering; rather, it is due to the bias in the observed average altering the values of the perturbations used to calculate instantaneous fluxes.

\section{d. Spectra}

Another consequence of advection by oscillating barotropic flow is the alteration of the spectra of the observed wave signal. In the case of no barotropic flow, the pure tone wave has energy only at a single frequency. As noted above, as $U / c$ increases, more wave crests are advected past the observing platform and energy appears at higher frequencies (Figure 5), but remains concentrated in harmonics of the fundamental frequency. This forces the observed signal to remain periodic over one wave period while allowing the observed waveform to take many shapes. For the $\mathrm{M}_{2}$ tide, as $U / c$ increases, more energy appears at the $\mathrm{M}_{4}, \mathrm{M}_{6}, \mathrm{M}_{8}, \ldots$ frequencies. The power spectra depend mostly on $U / c$; there is some spatial variability in the high-frequency content, but for a given $U / c$ only the highest frequency harmonics present are significantly affected by $\Phi_{0}$. The amplitude of the spectral peak at each harmonic varies with $U / c$. In general, less energy is found in lower harmonics as $U / c$ increases, but the amplitude of a given harmonic and the relative energies of any two harmonics are not simple functions of $U / c$ (Figure 6).

The results in MacKinnon and Gregg (2003) are consistent with these findings. They examined low-pass-filtered energy $\left(\mathrm{M}_{2}\right.$ and $\left.\mathrm{M}_{4}\right)$ in vertical modes 1 through 5 and found that, although a strong peak in $\mathrm{M}_{2}$ energy was present in the lowest modes, it was absent in the higher modes. For a given barotropic flow, higher-vertical mode waves have higher values of $U / c$ in general, and therefore will have less energy in lower harmonics. As the barotropic tidal amplitude increased, MacKinnon and Gregg (2003) found changes in the partitioning of energy between vertical modes, but no clear explanation for which modes had energy. This mirrors the results in Figure 6, which show the oscillations of first and second harmonic amplitudes at large values of $U / c$. 
The spectral energy contained in the observed signal, obtained by integrating the power spectra, is a function of both $U / c$ and $\Phi_{0}$ (Figure 7). For $U / c \sim 1.7$, the observed energy can range from 0.5 to 1.5 times the true energy of the wave, depending on $\Phi_{0}$. As $U / c$ increases, the sensitivity of the spectral energy to $\Phi_{0}$ decreases; this is because $\Phi_{0}$ affects only the amplitude of the few highest harmonics, so as energy is spread across more harmonics, $\Phi_{0}$ dependence decreases. Peaks in observed energy occur $\pi$ radians apart; the wavelength of the spectral energy is half that of the original wave. The spectral energy may correspond to kinetic or potential energy, depending on whether $u^{\prime}$ or $h^{\prime}$ is being measured. For a normal linear wave, these two quantities are $\pi / 2$ radians out of phase. The total observed energy (kinetic plus potential) is then constant, and any individual component (kinetic or potential energy) averaged over $1 / 2$ wavelength of the internal wave (neglecting $k_{0}$ ) will be constant. The periodic spatial variation in kinetic and potential energy resulting from the advection mechanism resembles a standing wave; indeed, it is possible that some features in shelf-seas with standing wave properties might be attributable to this interaction of the barotropic and baroclinic tides.

This spatial redistribution of kinetic and potential energy may help to explain the distribution of vertical mixing on the shelf. Dissipation measurements on the shelf contain many examples of "patchiness," with turbulence concentrated over a small horizontal extent (e.g., Inall et al., 2000), whereas Palmer et al. (2015) found links between dissipation and the ratio of kinetic to potential energy. If an internal wave is most likely to break at one point in its phase (e.g., when vertical shear is a maximum), partial stalling of the propagating zone of maximum IT shear by opposing barotropic flow will tend to spatially concentrate the zone of maximum shear at one location (for example, at $\mathrm{x}=0)$, while at the same time spatially diluting the shear at another location $(x \approx \lambda / 2$ in this example, where $\lambda$ is the wavelength of the IT). A similar mechanism may lead to spatial variability in bottom drag. The maximum flow speed over the seabed occurs where baroclinic ve- 
locity in the lower layer is in phase with the barotropic flow. Barotropic advection of the IT leads to spatial concentration of the higher bottom velocities, and may cause variability in bottom drag with a spatial scale $\sim \lambda$. The advection mechanism also leads to near-bed flows with higher harmonic frequencies; as these interact with sea floor topography, it may generate freely-propagating waves at the higher harmonics, similar to the processes described in detail by Bell (1976).

\section{Discussion}

\section{a. Implications for baroclinic energy fluxes}

The aforementioned shifts in observed energy, both spatially and towards higher frequencies, have important implications for estimates of baroclinic energy fluxes. The effects of advection by the barotropic tide introduce a potential source of variability to estimates whose magnitude depends in part on how data is collected and in part on how it is analyzed.

One approach to studying the internal tide is to use an array of moorings spanning the continental shelf (e.g., Hopkins et al., 2014). As we have demonstrated, where $U / c$ is large and in the absence of actual energy dissipation, the observed kinetic and potential energy of the internal tide will vary by up to $\pm 50 \%$ over one half-wavelength of the internal tide. If the phase offset between a locallygenerated internal tide and the local barotropic tide is constant, then placement of a mooring may bias observations of kinetic or potential energy by up to $\pm 50 \%$ (depending on $U / c$ ). Although the total energy (KE+PE) should remain constant, if moored instruments resolve one but not the other, it may introduce a bias into wave field estimates.

There are many sources of variability in the ocean, and identifying the variability associated with one particular process, such as the internal tide, is not easy. A standard analysis technique is to filter data using a low-pass or band-pass filter to selectively retain the processes of interest. 
Hopkins et al. (2014), for example, employed a band-pass filter to retain frequencies from 0.7 to $1.5 \mathrm{M}_{2}$. As we showed, however, the barotropic/baroclinic interaction shifts much of the observed energy into higher harmonics, even for reasonably small values of $U / c$. For any $U>0$, there is some reduction in the energy present in the $\mathrm{M}_{2}$ band. For $U / c \approx 0.5$, the observed energy at the $\mathrm{M}_{2}$ frequency ranges from 90 to $97 \%$ of its "true" value, whereas for $U / c=1$, it ranges from 65 to $88 \%$ of the actual energy (Figure $8 \mathrm{~b}$ ). Based on the barotropic velocities reported in several studies, and estimating the phase speed of the mode-1 internal tide, we can estimate by how much a particular reported baroclinic energy flux may be underestimated.

Another approach to reconstructing the wave field is to employ a harmonic fit to a signal of known frequency. Since the frequency of our model wave is partially shifted, problems arise. A harmonic fit to the observed wave in Equation 2 produces amplitude and phase estimates that vary with $U / c$ and $\Phi_{0}$ (Figure 8a,c). As with the mean in Figure 2, this implies spatial variability in the amplitude and phase of a harmonic fit. If $u^{\prime}$ and $p^{\prime}$ are fit to a harmonic, baroclinic energy fluxes scale as $A_{f i t}^{2}(\Phi)$, where $A_{f i t}$ is the amplitude of the harmonic fit. Variation of $A_{f i t}^{2}(\Phi)$ with $\Phi_{0}$ gives the appearance of horizontal divergence and convergence of energy flux. From such an observation, it would be natural to infer the existence of sinks (dissipation) or sources (local generation / tidal conversion) of energy. Figure 5 shows that the greatest energy flux divergence and convergence occurs when $U / c \approx 3.2$, where $F_{\text {observed }}$ ranges from $0.6 F$ to $1.3 F$ for an actual flux of $F$, with a second peak where $U / c \approx 1.4$, where $F_{\text {observed }}$ ranges from $0.5 F$ to $0.9 F$. The important point is that there are no such processes in our model. The apparent dissipation is in this case only an artifact of a low-pass filter applied to advected internal waves.

The variation of harmonic fit phase with $U / c$ is less important for baroclinic energy fluxes, but is relevant when considering the likelihood of local or remote generation of the internal tide, where 'local' implies constant phase offset relative to the barotropic tide and 'remote' is associated with 
a phase offset that changes in time (e.g., Nash et al., 2012). In our model, the phase of a harmonic fit to the observed waveform is a function of the phase offset and the normalized barotropic flow speed $U / c$ (Figure $8 \mathrm{c}$ ). If we consider the spring-neap cycle of the tides as a slowly-modulated tide with a frequency close to $\mathrm{M}_{2}$, then it is clear that in the ocean as spring tide approaches, the amplitude of the barotropic current $U$ increases, and therefore $U / c$ increases. This alters the phase of the least-squares fit solution to the observed wave. Therefore, even if the locally-forced internal tide has a constant phase offset relative to the local barotropic tide, the phase of the $\mathrm{M}_{2}$ harmonic fit to the observed internal tide will vary as $U / c$ increases. Similarly, as stratification changes, whether due to seasonal heating and cooling or a one-off mixing event, the internal wave speed will change, altering $U / c$. Changes in stratification also change the wavelength of the internal tide, which may alter its phase offset relative to the local barotropic tide by modifying $k_{n}$ in Equation 2 . These effects may lead to the locally-generated internal tide being at least partially miscategorized as remotely-generated when separating locally- and remotely-generated tides on the basis of local coherence.

Spring-neap changes in $U / c$ also affect the bias in observed baroclinic energy fluxes. With an averaging window long enough to capture changes in $U / c$, the observed bias will tend towards an average of the biases for the time-varying values of $U / c$. The variations in $U / c$ included in a longer time-averaging window will not drive the bias towards zero. However, with longer time windows, other ocean processes that affect $c$ or $\Phi_{0}$ may influence the energy flux bias in ways that are difficult to generalize.

In light of our results, a reexamination of baroclinic flux estimates in shelf seas may be needed. There are two ways to calculate baroclinic energy flux (Kunze et al., 2002). The first decomposes motions into barotropic and a baroclinic components. The perturbation velocity $\left(u^{\prime}\right)$ and pressure $\left(p^{\prime}\right)$ associated with an internal tide are used to calculate the baroclinic energy flux, defined by 
$F=\left\langle u^{\prime} p^{\prime}\right\rangle$, where $<>$ denotes a time average over a tidal cycle (Nash et al., 2005). This decomposition can be done in such a way as to eliminate certain influences of the barotropic tide, such as isopycnal heave caused by the motion of the free-surface (Kelly et al., 2010). However, these techniques do not correct for effects of the wave-wave interaction, caused by lateral advection of isopyncals by the barotropic tide.

The second approach measures the wave field, then calculates flux as $F=E c_{g}$, where $E$ is the energy in the internal tide and $c_{g}$ is the group velocity (See Inall et al., 2011 or Hopkins et al., 2014 for a more thorough discussion). It is clear that care must be taken in defining perturbation quantities, since spatial variability in the observed average is a consequence of advection by the barotropic tide. Inall et al. (2011) approached this problem with a towed undulator and found energy flux estimates of $940 \mathrm{~W} \mathrm{~m}^{-1}$ using $F=<u^{\prime} p^{\prime}>$ and $1600 \mathrm{~W} \mathrm{~m}^{-1}$ using $F=E c_{g}$. By averaging spatially over one baroclinic wavelength, they avoided the spatial bias in energy fluxes. By limiting the amount of filtering done, energy shifted to higher frequency contributed to the total, rather than being filtered out. It is not entirely surprising, therefore, that their across-shelf baroclinic energy flux estimates are much larger than others in the same region $\left(\mathrm{O}\left(100 \mathrm{~W} \mathrm{~m}^{-1}\right)\right)$ (Green et al., 2008; Hopkins et al., 2014).

One concern with the second approach $\left(F=E c_{g}\right)$ is the problem of partitioning energy between different vertical modes. The best estimate is of the form

$$
F=\sum_{i=1}^{\infty} E_{i} c_{i}
$$

Higher mode waves dissipate over shorter horizontal length scales than low modes, so we expect most of the energy away from generation sites to be in the lowest modes. However, the mode1 wave travels more quickly than other vertical modes, so assigning all baroclinic energy to the mode-1 wave is likely to overestimate the baroclinic energy. 
Both approaches may introduce errors into baroclinic energy flux divergence calculations, and therefore into indirect estimates of energy dissipation. A tempting solution, reconstruction of the 'unadvected' internal wave field, might be feasible in theory, but has been difficult to implement in practice. As Pinkel (2008) explains: "[Doppler smearing] cannot, in general, be unscrambled, but the task is much easier if the spectrum consists of a few discrete lines." This concords with our experience: methods that accurately reconstruct synthetic advected waves fail on real ocean data.

\section{b. Global applicability}

Tidal energy conversion is directly proportional to $U$, and barotropic velocities generally increase as water depth decreases, whereas baroclinic wave speeds decrease. Therefore, we expect that the bias presented here will be present at most shallow internal tide generation sites and will be most pronounced in shelf seas with strong barotropic tides. To estimate the parameter $U / c$, we first calculated dynamical mode estimates of the mode-1 internal wave speed for the $\mathrm{M}_{2}$ internal tide by solving the wave equation,

$$
\frac{\partial^{2} \eta}{\partial z^{2}}+\left(\frac{N^{2}-\omega^{2}}{\omega^{2}-f^{2}}\right) k^{2} \eta=0
$$

where $N$ is the buoyancy frequency, $\omega$ is the wave frequency, $f$ is the inertial frequency, and $k$ is the horizontal wave number of the internal wave. Stratification profiles were derived from long-term average temperature and salinity profiles from the World Ocean Atlas (https://www.nodc.noaa.gov/OC5/woa13/) (Locarnini et al., 2013; Zweng et al., 2013). Then using tidal velocities from TPXO (http://volkov.oce.orst.edu/tides/global.html) (Egbert and Erofeeva, 2002), we estimate $U / c$ for the first baroclinic mode. The results of the computation, shown in Figure 9, show that the ratio is greatest $(\mathrm{O}(10))$ on the European Shelf. Elevated values are also evident east of Argentina, northwest of Australia, in the South China Sea, and on the New England 
Shelf. Overall, these calculations indicate potential for $U / c>1$ in $\sim 3.5 \%$ of the ocean, or more than one third of the ocean shallower than $500 \mathrm{~m}$. However, these measurements do not account for the relative directions of wave propagation and barotropic flow; internal tides propagating at angles to the semimajor axis of the barotropic tidal ellipses will have smaller values of $U / c$. Furthermore, seasonal variations in stratification will affect the values of $c ; U / c$ is likely to be higher in winter than summer, especially in shelf seas where the annual cycle in stratification is large. Small values of $U / c$ over the deep ocean mean that the abyssal ocean is unaffected by any bias.

Using the values of $U / c$ in Figure 9, we calculate an 'underestimation factor' for baroclinic energy fluxes; this is the 'worst-case' estimate for how much a linear internal tide may be underestimated using stationary sampling techniques and harmonic fits (the narrowest spectral filter), based on barotropic tidal advection of the baroclinic tide (see Figure 10 for details). We now apply this estimate to a baroclinic energy flux estimate in the Celtic Sea. At their mooring ST4, moored in $\sim 160 \mathrm{~m}$ deep water on the continental shelf $40 \mathrm{~km}$ shoreward the shelf break, Hopkins et al. (2014) reported total average onshelf baroclinic fluxes of $93 \mathrm{~W} \mathrm{~m}^{-1}$, and average semidiurnal onshelf baroclinic energy fluxes of $28 \mathrm{~W} \mathrm{~m}^{-1}$, a phase speed of $0.5-0.6 \mathrm{~m} / \mathrm{s}$ for the mode- 1 baroclinic tide, and maximum on-shelf barotropic currents of $\sim 0.4 \mathrm{~m} \mathrm{~s}^{-1}$. Here $U / c$ is $\sim 0.7-0.8$. Referring to Figure 4, we estimate that the observed, unfiltered energy fluxes may represent as little as $75 \%$ of the total baroclinic energy fluxes present. Figure 8a indicates that the filtered amplitude of the observed internal tide ranges from 0.78 to 0.9 times its actual value. The amplitudes of the filtered baroclinic energy fluxes are calculated by squaring two filtered values, so the observed fluxes likely range from $\sim 0.6-0.8$ times their actual value. In other words, the real values are estimated to be $25-67 \%$ higher. Computing empirical orthogonal functions (EOFs) of the across-shelf velocity, Hopkins et al. (2014) found that the mode-1 EOF accounted for $45 \%$ of the variance in the bandpassed across-shelf velocity fields, while the mode- 2 EOF accounted for $11-16 \%$ of 
the variance. With a phase speed of $0.3 \mathrm{~m} / \mathrm{s}$ for the slower mode- 2 baroclinic tide, $U / c$ has a value of $\sim 1.3$. This leads to unfiltered baroclinic energy flux estimates that capture only $55-85 \%$ of the total energy flux (as in Figure 4). With the higher mode wave, more baroclinic energy is shifted to higher harmonics that are filtered out before energy fluxes are calculated; the observed fluxes in the filtered data are only 0.2-0.6 times the "real" baroclinic energy fluxes, making the total values $67-400 \%$ larger. However, this and higher modes contain much less energy than the first mode. To evaluate the amount by which the filtered baroclinic energy fluxes underestimate the total, we need estimates of the modal distribution of energy fluxes. We assume the fraction of energy contained in a baroclinic mode is comparable to the fraction of variance explained by EOFs of baroclinic velocity in Hopkins et al (2014). For modes 1 and 2, we take these to be $45 \%$ and $16 \%$, respectively, and add a correction for each mode based on the value of $U / c$ for that mode, as calculated above. The lower and upper bounds on the correction needed are as follows: mode 1: $(25$ to $67 \%$ increase needed $) \times 45 \%$ of baroclinic energy + mode $2:(67$ to $400 \%$ increase $) \times$ $16 \%$ of baroclinic energy, all divided by $(45 \%+16 \%)$, the fraction of baroclinic energy in modes 1 and 2. The result is that the filtered baroclinic energy fluxes should be between $36 \%$ and $150 \%$ larger than the original estimates. That is, the original estimates represent $\sim 40-73 \%$ of the energy flux. Rather than accounting for only $30 \%$ of the total baroclinic energy fluxes $\left(28 \mathrm{~W} \mathrm{~m}^{-1}\right.$ out of $93 \mathrm{~W} \mathrm{~m}^{-1}$ ), the semidiurnal internal tide is likely responsible for $40-75 \%$ of the total. Meanwhile, the original estimated total (unfiltered) baroclinic energy estimates likely captured 68-96\% of the total baroclinic energy flux. The revised estimate of the total baroclinic energy flux ranges from $97-136 \mathrm{~W} \mathrm{~m}^{-1}$.

Studies in other shelf seas that have computed baroclinic energy fluxes using low-pass filters, bandpass filters, or harmonic fits have likely underestimated baroclinic energy fluxes in similar fashion. The information in Figure 4 and Figure 10 may be seen as first-order correction factors 
that could be employed a posteriori to improve computations from any area. However, since tidal amplitude $U$ changes over a spring-neap cycle and wave phase speed $c$ changes with stratification, a more accurate estimate of the correction factor requires more specific values of $U$ and $c$, rather than the values based on long-term averages. With a specific value of $U / c$, the upper and lower bounds on the underestimation can be inferred by reference to Figure 8a.

\section{Conclusions}

In this paper, we have explored a very simple model of a baroclinic tide advected by oscillating barotropic flow. Despite its simplicity, the model replicates many of the unusual features of the internal tide in shelf seas: unusual wave forms, high-frequency energy, and spatial and temporal variability in the phase and amplitude of the baroclinic tide. Our results suggest that advection of the internal tide by the barotropic tide biases observed average quantities, such as pressure and baroclinic velocity and hence also affect the perturbation quantities. As a result, baroclinic energy fluxes appear to be larger and more intermittent than they would in the absence of advection. These confounding factors make analysis of the internal tide more difficult, and expose a need for great care in analysing the internal tide in shelf seas. Neglecting this process where it is important can easily lead to a significant underestimate of the strength of baroclinic energy fluxes. On the other hand, although this barotropic/baroclinic interaction may lend the appearance of randomness to a well-ordered internal tide, it introduces the possibility that mechanisms governing temporal and spatial variability in internal tides may be less complicated than has been thought.

Our results suggest that correcting for the low bias in energy flux estimates in the Celtic Sea (Hopkins et al., 2014) may significantly increase estimates of total baroclinic energy fluxes, and will also increase the proportion of baroclinic energy fluxes attributed to the semidiurnal tide. In cases where data are strongly filtered, the increase can be a factor of 2 to 3 . The adjustments 
we have applied are fairly crude, however, and they do not close the gap in Celtic Sea baroclinic energy fluxes. Accounting for and correcting the biases in various quantities, from, for example, average thermocline displacement (Section 3.2) or baroclinic energy flux magnitude (Section 3.5) will require some effort, and may change how we understand other shelf break processes. This process is likely to be significant in many other shelf seas, but the global significance of the upward adjustment we project in shelf seas baroclinic energy fluxes remains a subject of inquiry.

Acknowledgments. Funding was provided by NERC through FASTNEt (grant NE/I030224/1). The authors wish to acknowledge M. J. Lewis for useful discussions and three anonymous reviewers for their helpful and insightful suggestions. 


\section{References}

Bell, T. J., 1976: The structure of internal wave spectra as determined from towed thermistor chain measurements. J. Geophys. Res., 81, 3709=3714, doi:10.1029/JC081i021p03709.

Egbert, G., and S. Erofeeva, 2002: Efficient inverse modeling of barotropic ocean tides. J. Atmos.

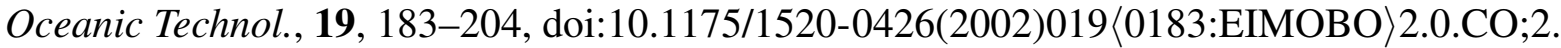

Green, J., and J. Nycander, 2013: A comparison of tidal conversion parameterizations for tidal models. J. Phys. Oceanogr., 43, 104-119, doi:10.1175/JPO-D-12-023.1.

Green, J., J. Simpson, S. Thorpe, and T. P. Rippeth, 2010: Observations of internal tidal waves in the isolated seasonally stratified region of the western Irish Sea. Continental Shelf. Res., 30, 214-225, doi:10.1016/j.csr.2009.11.004.

Green, J. A. M., J. H. Simpson, S. Legg, and M. R. Palmer, 2008: Internal waves, baroclinic energy fluxes, and mixing at the European shelf edge. Continental Shelf Research, 28, 937-950, doi:10.1016/j.csr.2008.01.014.

Holloway, G., 1983: A conjecture relating oceanic internal waves and small-scale processes. Atmosphere-Ocean, 21 (1), 107-122, doi:10.1080/07055900.1983.9649159.

Hopkins, J. E., G. R. Stephenson, J. A. M. Green, and M. E. Inall, 2014: Storms modify baroclinic energy fluxes in a seasonally stratified shelf sea: inertial-tidal interaction. J. Geophys. Res., 119, 6863-6883, doi:10.1002/2014JC010011.

Inall, M., D. Aleynik, T. Boyd, M. Palmer, and J. Sharples, 2011: Internal tide coherence and decay over a wide shelf sea. Geophys. Res. Lett., 38 (L23607), doi:10.1029/2011GL049943.

Inall, M. E., T. P. Rippeth, and T. J. Sherwin, 2000: Impact of nonlinear waves on the dissipation of internal tidal energy at a shelf break. J. Geophys. Res., 105, doi:8687-8705. 
Kelly, S., J. Nash, and E. Kunze, 2010: Internal-tide energy over topography. J. Geophys. Res., 115, doi:10.1029/2009JC005618.

Kelly, S. M., N. L. Jones, J. D. Nash, and A. F. Waterhouse, 2013: The geography of semidiurnal mode-1 internal-tide energy loss. Geophys. Res. Lett., 40, 4689-4693, doi:10.1002/grl.50872.

Kunze, E., L. Rosenfeld, G. Carter, and M. Gregg, 2002: Internal waves in Monterey Submarine Canyon. J. Phys. Oceanogr., 32, 18901913.

Locarnini, R. A., and Coauthors, 2013: World Ocean Atlas 2013, Volume 1: Temperature, Levitus, S. Ed., A. Mishonov Technical Ed. (NOAA Atlas NESDIS 73), 40 pp.

MacKinnon, J. A., and M. Gregg, 2003: Shear and baroclinic energy flux on the summer New England shelf. J. Phys. Oceanogr., 33, 1462-1475.

Melet, A., R. Hallberg, S. Legg, and K. Polzin, 2013: Sensitivity of the ocean state to the vertical distribution of internal-tide driven mixing. J. Phys. Oceanogr., 43, 602-615, doi: 10.1175/JPO-D-12-055.1.

Nash, J., M. Alford, and E. Kunze, 2005: Estimating internal wave energy fluxes in the ocean. $J$. Atmos. Oceanic Tech., 22, 1551-1570, doi:10.1175/JTECH1784.1.

Nash, J., S. Kelly, E. Shroyer, J. Moum, and T. Duda, 2012: The unpredictable nature of internal tides on continental shelves. J. Phys. Oceanogr., 42, 1981-2000, doi:10.1175/JPO-D-12-028.1.

Osborne, A. R., T. L. Burch, and R. I. Scarlet, 1978: The influence of internal waves on deep-water drilling. J. Petrol. Technol., 30, 1497-1504.

Palmer, M., G. R. Stephenson, M. E. Inall, C. Balfour, A. Dusterhus, and J. A. M. Green, 2015: Turbulence and mixing by internal waves in the Celtic Sea determined from ocean glider microstructure measurements. J. Marine Systems, 144, 7-69. 
Pinkel, R., 2008: Advection, phase distortion, and the frequency spectrum of finescale fields in the sea. J. Phys. Oceanogr., 38, 291-313, doi:10.1175/2007JPO3559.1.

Rippeth, T., and M. Inall, 2002: Observations of the internal tide and associated mixing across the Malin Shelf. J. Geophys. Res., 107, 11 980-11 990, doi:10.1029/2000JC000761.

Robins, P., and A. J. Elliott, 2009: The internal tide of the Gareloch, a Scottish fjord. Estuarine, Coastal and Shelf Science, 81, 130-142.

Sharples, J., C. Moore, and E. Abraham, 2001: Internal tide dissipation, mixing, and vertical nitrate flux at the shelf edge of NE New Zealand. J. Geophys. Res., 106, 14 069-14 081.

Sharples, J., and Coauthors, 2007: Spring-neap modulation of internal tide mixing and vertical nitrate fluxes at a shelf edge in summer. Limnol. Oceanogr., 52, 1735-1747.

Shroyer, E., J. N. Moum, and J. Nash, 2011: Nonlinear internal waves over New Jersey's continental shelf. J. Geophys. Res., 116 (C03022), doi:10.1029/2010JC006332.

Stephenson, G. R. J., J. E. Hopkins, J. A. M. Green, M. E. Inall, and M. R. Palmer, 2015: Windmixing by storms modifies baroclinic energy flux on the Celtic Sea shelf. Geophys. Res. Lett., doi:10.1002/2014GL062627.

Vlasenko, V., N. Stashchuk, M. Inall, and J. E. Hopkins, 2014: Tidal energy conversion in a global hot spot: on the 3d dynamics of baroclinic tides at the Celtic Sea shelf break. J. Geophys. Res., doi:10.1002/2013/C009708.

Wunsch, C., and R. Ferrari, 2004: Vertical mixing, energy, and the general circulation of the oceans. Annu. Rev. Fluid Mech., 36, 281-314, doi:10.1146/annurev.fluid.36.050802.122121.

Zweng, M., and Coauthors, 2013: World Ocean Atlas 2013, Volume 2: Salinity, Vol. 74. NOAA Atlas NESDIS, 39 pp. pp. 


\section{LIST OF FIGURES}

Fig. 1. Observed waves (black) as in Equation 2 and least-squares sinusoidal fit to the observed wave (red) for $\frac{U}{c}=1.7$ and phase offsets a) $\Phi_{0}=0$, b) $\Phi_{0}=\frac{\pi}{3}$, c) $\Phi_{0}=\frac{2 \pi}{3}$, d) $\Phi_{0}=\pi$, e) $\Phi_{0}=\frac{4 \pi}{3}$, and f) $\Phi_{0}=\frac{5 \pi}{3}$. Dashed lines indicate the mean value of the observed wave,

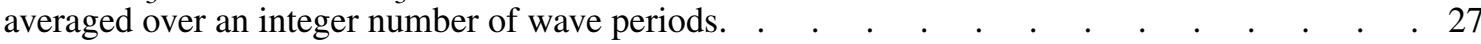

Fig. 2. The mean of the observed wave, as in Equation 2, as a function of $\Phi_{0}$ and $U / c . \quad$. . . . . . 28

Fig. 3. Baroclinic energy flux, $F=u^{\prime} p^{\prime}$, where $u^{\prime}$ and $p^{\prime}$ are defined using Equation 2 with $\mathrm{A}=1$ and with the time-mean removed. a) Timeseries of $F$ for $\frac{U}{c}=0, \Phi_{0}=\frac{\pi}{2}$ (green), $\frac{U}{c}=1.7$, $\Phi_{0}=\frac{\pi}{2}$ (red), and $\frac{U}{c}=1.7, \Phi_{0}=\pi$ (blue). Similar plots for a range of $\Phi_{0}$ with b) $\frac{U}{c}=1.7$, and c) $\frac{U}{c}=0.2$.

Fig. 4. Tidally-averaged baroclinic energy flux $\langle F\rangle=\left\langle u^{\prime} p^{\prime}\right\rangle$, where $u^{\prime}$ and $p^{\prime}$ are defined using Equation 2 with $\mathrm{A}=1$ and with the time-mean removed. $\langle F\rangle$ is further normalized by the case where $U / c=0$. Average fluxes vary with $\Phi_{0}$, but generally reach a minimum where $U / c \sim 2$ and a maximum where $U / c \sim 3$.

Fig. 5. Power spectra against frequency (y-axis) of the wave in Equation 2 as a function of $\frac{U}{c}$. Spectra have been averaged over $\Phi_{0} \in[0,2 \pi)$. As $\frac{U}{c}$ increases, more energy is present at higher harmonics.

Fig. 6. Normalized spectral amplitude at harmonics of the $\mathrm{M}_{2}$ tide for a freely propagating sine wave advected by sinusoidally oscillating flow of the same frequency, where the advective velocity, $U$, has been normalized by the speed of the wave, $c$.

Fig. 7. The integral of the power spectra of the observed wave (as in Equation 2) over one wave period, normalized to 1 when $U=0$. Observed signal power varies with $\frac{U}{c}$ and $\Phi_{0}$. . . . . . . 33

Fig. 8. a) The maximum (red) and minimum (blue) amplitude of a harmonic fit to the advected wave, as a function of $U / c$. The b) amplitude and c) phase of harmonic fits to the advected wave vary with $U / c$ and $\Phi_{0}$. Contours are spaced 0.2 units apart. . . . . . . . . . . . . . 34

Fig. 9. Global map of $\frac{U}{c}$ for (i) $\mathrm{M}_{2}$ and (ii) $\mathrm{K}_{1}$ tidal constituents. A mask (gray) has been applied over land surfaces and poleward of the critical latitude for each tidal frequency.

Fig. 10. Based on $\frac{U}{c}$ in Figure 9, the factor by which a harmonic fit (the most restrictive spectral filter) will underestimate an advected internal wave amplitude. For example, a value of 0.2 means that the amplitude of the harmonic fit is $20 \%$ the amplitude of the advected IW. 


\section{a)}

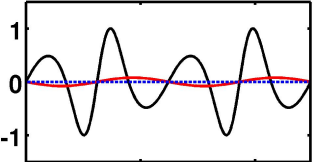

c)

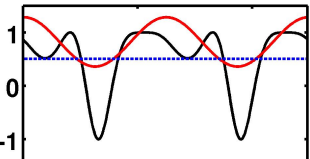

e)

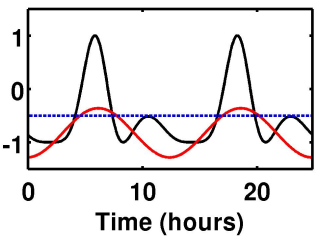

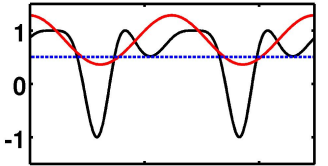

d)

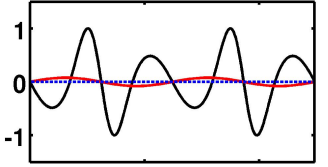

f)

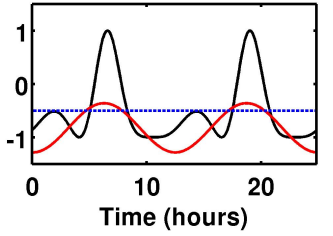

FIG. 1. Observed waves (black) as in Equation 2 and least-squares sinusoidal fit to the observed wave (red) for $\frac{U}{c}=1.7$ and phase offsets a) $\Phi_{0}=0$, b) $\Phi_{0}=\frac{\pi}{3}$, c) $\Phi_{0}=\frac{2 \pi}{3}$, d) $\Phi_{0}=\pi$, e) $\Phi_{0}=\frac{4 \pi}{3}$, and f) $\Phi_{0}=\frac{5 \pi}{3}$. Dashed lines indicate the mean value of the observed wave, averaged over an integer number of wave periods. 


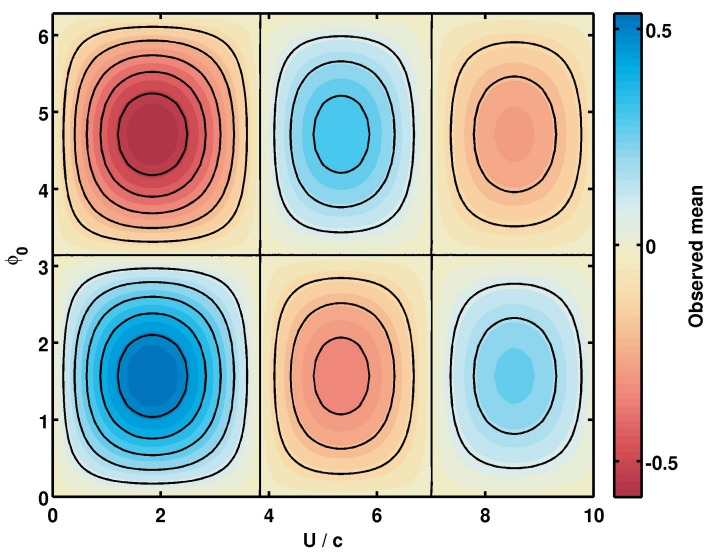

FIG. 2. The mean of the observed wave, as in Equation 2, as a function of $\Phi_{0}$ and $U / c$. 

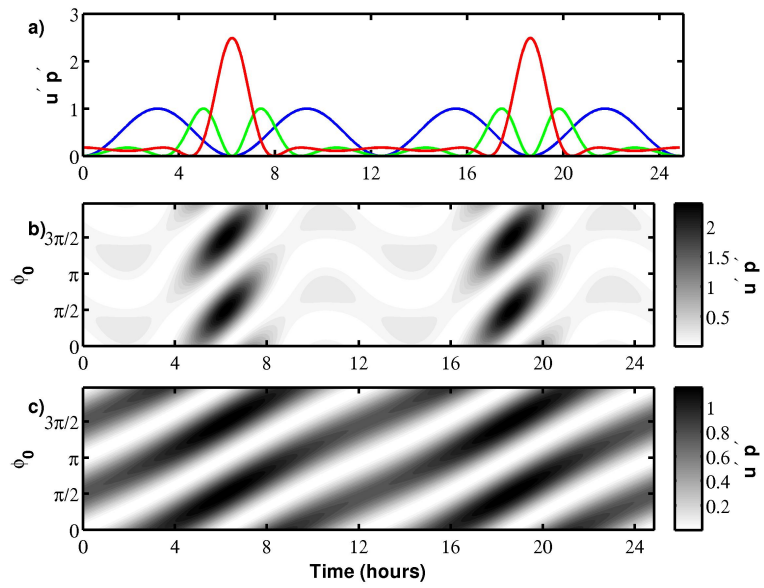

FIG. 3. Baroclinic energy flux, $F=u^{\prime} p^{\prime}$, where $u^{\prime}$ and $p^{\prime}$ are defined using Equation 2 with $\mathrm{A}=1$ and with the time-mean removed. a) Timeseries of $F$ for $\frac{U}{c}=0, \Phi_{0}=\frac{\pi}{2}$ (green), $\frac{U}{c}=1.7, \Phi_{0}=\frac{\pi}{2}$ (red), and $\frac{U}{c}=1.7$, 557 $\Phi_{0}=\pi$ (blue). Similar plots for a range of $\Phi_{0}$ with b) $\frac{U}{c}=1.7$, and c) $\frac{U}{c}=0.2$. 


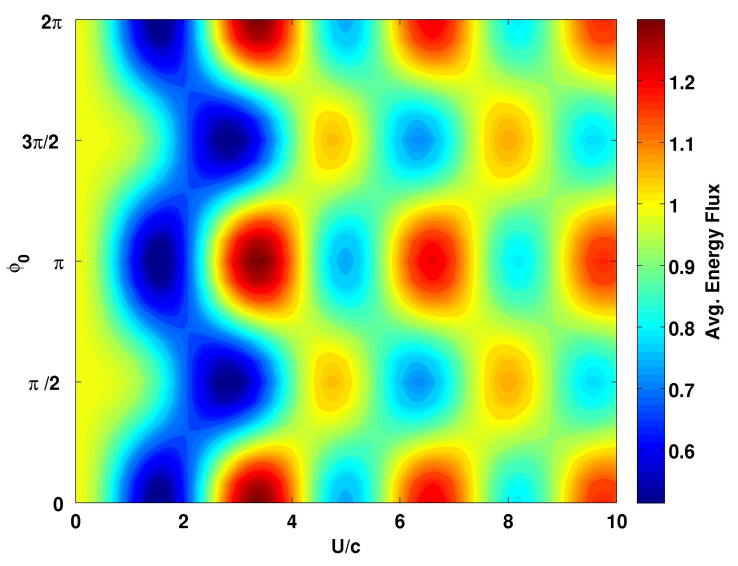

FIG. 4. Tidally-averaged baroclinic energy flux $\langle F\rangle=\left\langle u^{\prime} p^{\prime}\right\rangle$, where $u^{\prime}$ and $p^{\prime}$ are defined using Equation 2 with $\mathrm{A}=1$ and with the time-mean removed. $\langle F\rangle$ is further normalized by the case where $U / c=0$. Average

fluxes vary with $\Phi_{0}$, but generally reach a minimum where $U / c \sim 2$ and a maximum where $U / c \sim 3$. 


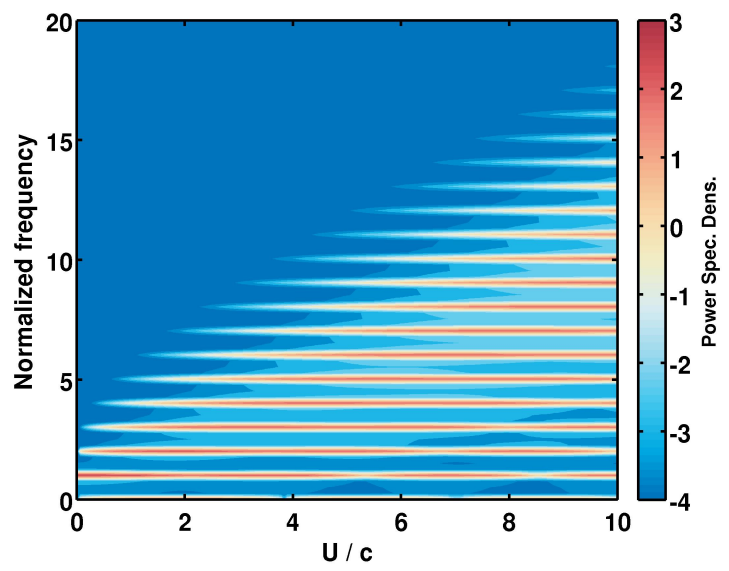

${ }_{561}$ FIG. 5. Power spectra against frequency (y-axis) of the wave in Equation 2 as a function of $\frac{U}{c}$. Spectra have 562 been averaged over $\Phi_{0} \in[0,2 \pi)$. As $\frac{U}{c}$ increases, more energy is present at higher harmonics. 


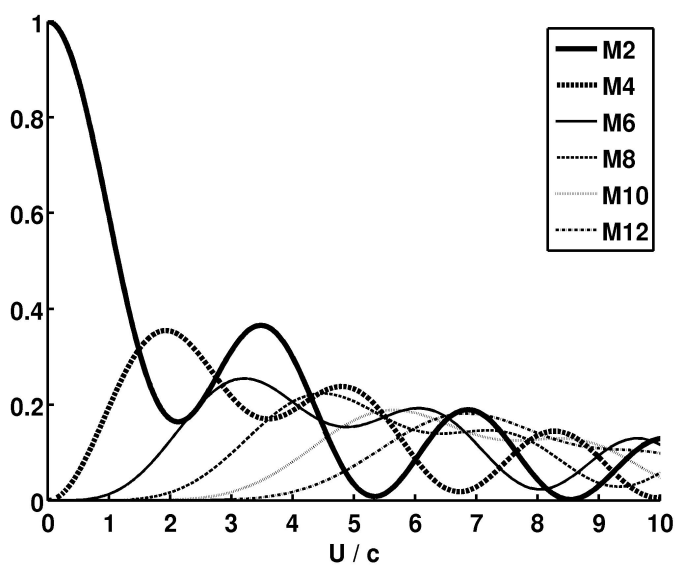

FIG. 6. Normalized spectral amplitude at harmonics of the $\mathrm{M}_{2}$ tide for a freely propagating sine wave advected by sinusoidally oscillating flow of the same frequency, where the advective velocity, $U$, has been normalized by the speed of the wave, $c$. 


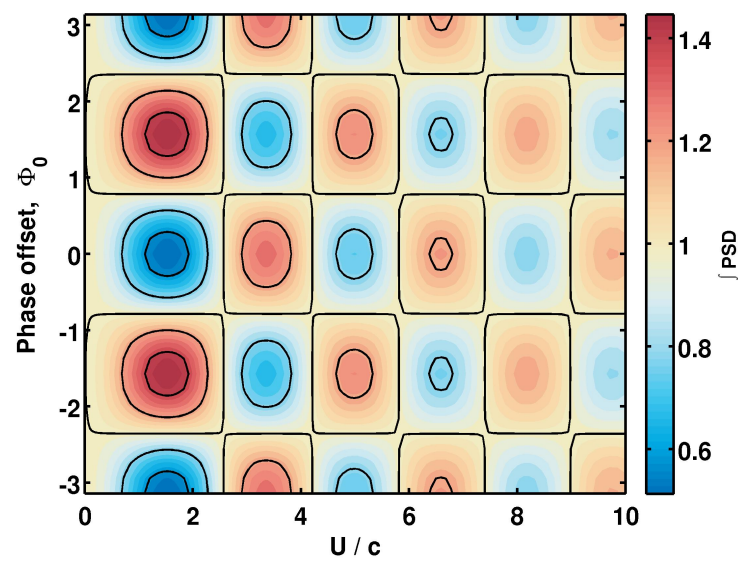

566 FIG. 7. The integral of the power spectra of the observed wave (as in Equation 2) over one wave period, ${ }_{567}$ normalized to 1 when $U=0$. Observed signal power varies with $\frac{U}{c}$ and $\Phi_{0}$. 

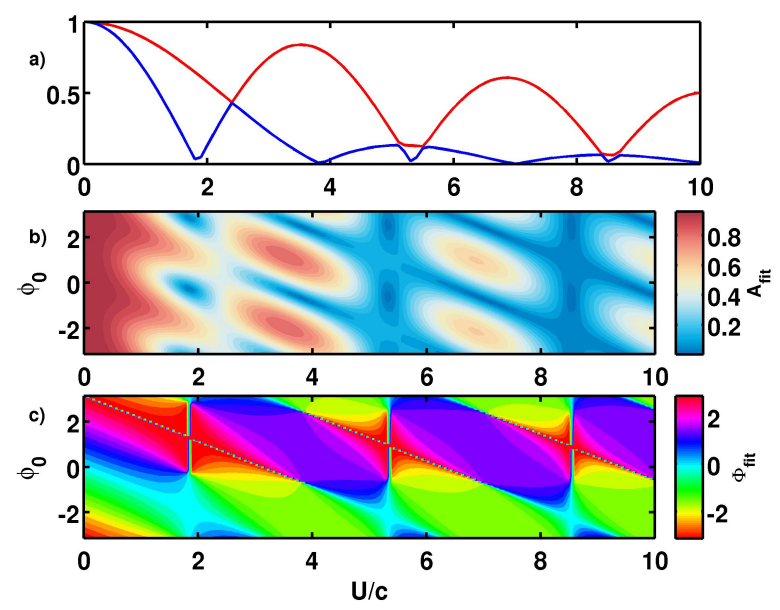

FIG. 8. a) The maximum (red) and minimum (blue) amplitude of a harmonic fit to the advected wave, as a 569 function of $U / c$. The b) amplitude and c) phase of harmonic fits to the advected wave vary with $U / c$ and $\Phi_{0}$. 570 Contours are spaced 0.2 units apart. 

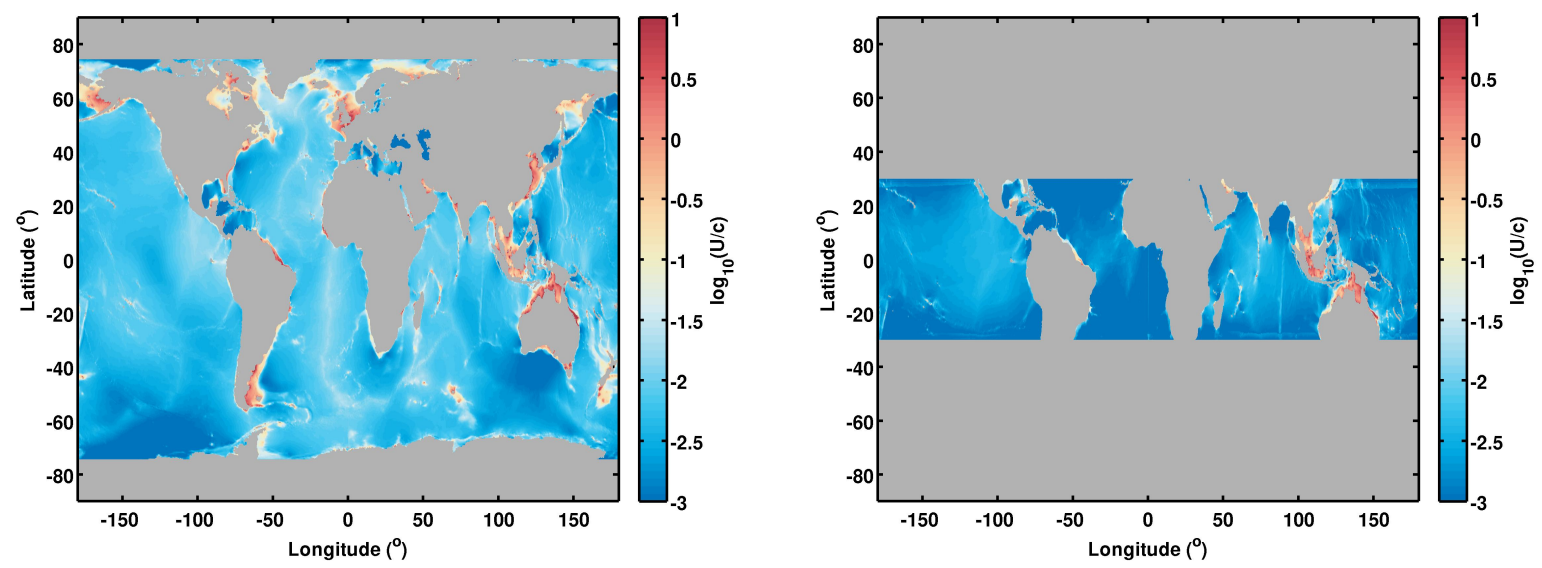

FIG. 9. Global map of $\frac{U}{c}$ for (i) $\mathrm{M}_{2}$ and (ii) $\mathrm{K}_{1}$ tidal constituents. A mask (gray) has been applied over land 572 surfaces and poleward of the critical latitude for each tidal frequency. 


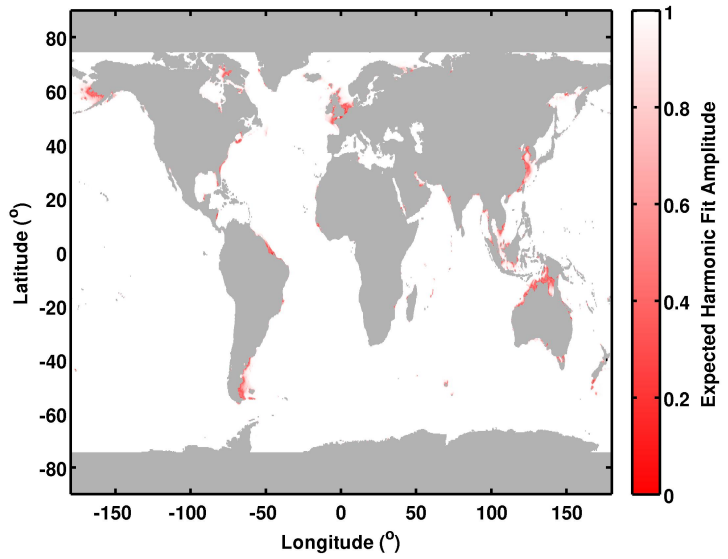

573 FIG. 10. Based on $\frac{U}{c}$ in Figure 9, the factor by which a harmonic fit (the most restrictive spectral filter) will underestimate an advected internal wave amplitude. For example, a value of 0.2 means that the amplitude of the 575 harmonic fit is $20 \%$ the amplitude of the advected IW. 